\title{
GLIDING HUMP PROPERTIES AND SOME APPLICATIONS
}

JOHANN BOOS

JOHANN BOOS

lachberrich Mathematik

l.nnlinivenitä (iesamthochschule

I) $580 \times 4$ Hagen

(iermany
DANIEL J. FLEMING

DANIEL J. FLEMING

Department of Mathematics

St. Lawrence Iniversity

Canton, NY 13617

ISA

(Received July 21, 1993)

ABSTRACT. In this note we consider several types of gliding hump properties for a sequence space $E$ and we consider the various implications between these properties. By means of examples we show that most of the implications are strict and they afford a sort of structure between solid sequence spaces and those with weakly sequentially complete $\beta$-duals. Our main result is used to extend a result of Bennett and kalton which characterizes the class of sequence spaces $E$ with the property that $E \subset S_{F}$, whenever $F$ is a separable $\mathrm{FK}$ space containing $E$ where $S_{F}$ denotes the sequences in $F$ having sectional convergence. Ihis, in turn, is used to identify a gliding humps property as a sufficient condition for $E$ to be in this class.

KEY WORDS AND PHRASES. Gliding hump properties, weak sequential completeness of the $\beta$-dual, sectional convergence in FK spaces, Theorem of Schur, Theorem of Hahu.

AMS 1980 CLASSIFICATION CODES. 46A45, 46A35, 40D25, $40 \mathrm{H} 05$.

\section{INTRODUCTION.}

Over the past eighty years the "gliding hump" technique has been a frequently used tool to establish results in summability and sequence space theory. Among the more familar examples would be the Silverman-Toeplitz theorem which gives necessary and sufficient conditions for the regularity of a summability method [22], the Mazur-Orlicz bounded consistency theorem ([6], [12] and [13]), the theorem of Köthe and Toeplitz on the weak sequential completeness of the Köthe dual of a solid sequence space [11] and the theorems of Schur on the characterization of coercive matrices and the equivalence of weak and strong convergence in $\ell_{1}[19]$. Whereas the first three of these have subsequently been argued using functional analytic techniques (see e.g. [24] and [10]) no such "soft" proofs of Schur's theorems are known. Various authors have considered sequence spaces enjoying certain gliding hump type properties. See for example, [8] for extensions of Schur's theorems, ([4], [5], [20]) for Mazur -Orlicz type theorems and ([5], [14]) for weak sequential completeness results. The gliding hump technique has also proven to be a key ingredient in the solution to problems related to the Wilansky Property ([1], [21], [15]).

In section 3 of this note we introduce various types of gliding hump properties and discuss the implications between them. We give examples in section 5 to show that most of these implications are strict and they are, in some sense, affording a structure to the set of sequence spaces between the solid spaces and those with weakly sequentially complete $\beta$-duals. 


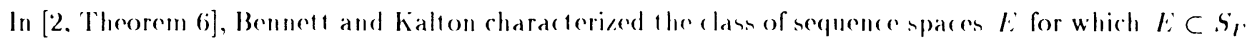
whenever $F$ is a separable $F \mathrm{~h}$ space containing $E$ (here, $S_{F}$ denoles the elements of $F$ having sectional convergence). In Theorem 3.6 we extend their result by showing that it suflices to consider only the case where $F$ is a convergence domain of a matrix. Combining this observation with our main result Theorem 3.5 we obtain in corollary 3.7 the more tractable pointuise weak gliding hump property (see definiton 3.1 below) as a sufficient condition for $E$ to belong to this class. In section 1 we apply the techniques of this paper to obtain short proofs of some classical results.

\section{NOTATION AND PRELIMINARIFS.}

Let $\omega$ denote the linear space of all scalar (real or complex) sequences. By a sequence space $l:$ we shall mean any linear subspace of $\omega$. A sequence space $E$ endowed with a locally convex topology is called a $K$-space if the inclusion map $\imath: E \longrightarrow \omega$ is continuous where $\omega$ has the topology of coordinatewise convergence. $\Lambda K$-space $E$ with a Fréchet topology is called an $F K$-space. If, in addition, the topology is normable then $E$ is called a $B K^{-}$-space. We assume throughout this note familarity with the standard sequence spaces and their natural topologies (see e. g. [24], [9]).

For a sequence space $E$ the multiplier space of $E$ and the $\beta$-dual of $E$ are given by

$$
\mathcal{M}(E)=\{x \in \omega \mid x y \in E \text { for each } y \in E\}
$$

and

$$
E^{\beta}=\left\{x \in \omega \mid \sum_{k} x_{k} y_{k} \text { converges for each } y \in E\right\}
$$

where $x y$ denotes the coordinatewise product. For $x \in \omega, n \in \mathbb{N}$ the $n^{\text {th }}$ section of $x$ is

$$
x^{[n]}=\sum_{k=1}^{n} x_{k} e^{k}
$$

where $e^{k}=\left(\delta_{i k}\right)_{i=1}^{\infty}$ is the $k^{\text {th }}$ coordinate vector. For any positive term sequence $\mu=\left(\mu_{k}\right)$ let

$$
E_{\mu}=\left\{x \in \omega \mid\left(\frac{x_{k}}{\mu_{k}}\right) \in E\right\} .
$$

If $(E, F)$ is a dual pair then $\sigma(E, F), \tau(E, F)$ denotes the weak topology and the Mackey topology respectively. For a sequence space $E$ and a linear subspace $F$ of $E^{\beta},(E, F)$ is a dual pair under the natural bilinear form

$$
\langle x, y\rangle=\sum_{k} x_{k} y_{k}
$$

If $E$ is a $K$-space containing $\varphi$, the space of finitely non-zero sequences, we let

$$
\begin{aligned}
& L_{E}=\left\{x \in E \mid\left\{x^{[n]} \mid n \in \mathbb{N}\right\} \text { is bounded in } E\right\} \\
& W_{E}=\left\{x \in E \mid x^{[n]} \longrightarrow x \quad \sigma\left(E, E^{\prime}\right)\right\} \\
& S_{E}=\left\{x \in E \mid x^{[n]} \longrightarrow x \text { in } E\right\}
\end{aligned}
$$

where $E^{\prime}$ denotes the topological dual of $E$. A $K$-space $E$ containing $\varphi$ with $E=S_{E}$ is called an $A K$-space.

If $A=\left(a_{n k}\right)$ is an infinite matrix with scalar entries the convergence domain

$$
c_{A}=\left\{x \in \omega \mid A x=\left(\sum_{k} a_{n k} x_{k}\right)_{n=1}^{\infty} \in c\right\}
$$


admits a natural $l \mathrm{~K}$ topology [21]. For $r \in r_{A}$ we write lim, $x=\lim \mathbf{1} x$.

If $\varphi \subset c_{A}$ let $a_{k}=\lim _{n} a_{n k}$ and define

$$
I_{A}=\left\{r \in A_{A} \mid \sum_{k} a_{k} \cdot r_{k} \text { exist }\right\},
$$

$\Lambda_{A}: I_{A} \longrightarrow \mathbb{K}$ by $\Lambda_{A}(x)=\lim _{A} x-\sum_{k} a_{k} x_{k}($ where $\mathbb{K}=\mathbb{C}$ or $\mathbb{K}=\mathbb{R})$ and

$$
\Lambda_{A}^{\perp}=\left\{x \in I_{A} \mid \Lambda_{A}(x)=0\right\} .
$$

Further if $\varphi \subset c_{A}$ we write $L_{A}, W_{A}, S_{A}$ instead of $L_{r_{A}}, W_{r_{A}}, S_{c_{A}}$. In this case $W_{A}^{\prime}=L_{A} \cap \Lambda_{A}^{\perp}$ (sec c. g. $[24])$.

\section{THE GIIINING IIUMP PROPERTIES.}

We begin by introducing several types of gliding hump properties.

DEFINITION 3.1. A sequence $\left(y^{(n)}\right)$ in $\omega \backslash\{0\}$ is called a block sequence if there exists an index sequence $(k$,$) such that y_{k}^{(n)}=0$ for any $n, k \in \mathbb{N}$ with $\left.\left.k \notin\right] k_{n-1}, k_{n}\right]$ where $k_{0}:=0$, and it is called a 1-block sequence if furthermore $y_{k}^{(n)}=1$ for each $\left.\left.k \in\right] k_{n-1}, k_{n}\right]$ and $n \in \mathbb{N}$.

Let $E$ be a sequence space containing $\varphi$.

- $E$ has the gliding hump property (ghp) if for each block sequence $\left(y^{(n)}\right)$ satisfying $\sup _{n \in \mathbb{N}}\left\|y^{(n)}\right\|_{b v}<\infty$ and any monotonicly increasing sequence $\left(n_{k}\right)$ of integers there exists a subsequence $\left(m_{k}\right)$ of $\left(n_{k}\right)$ with $\sum_{j=1}^{\infty} y^{\left(m_{\jmath}\right)} \in E$ (pointwise sum).

- $E$ has the pointwise gliding hump property (p ghp) if for each $x \in E$, any block sequence $\left(y^{(n)}\right)$ satisfying $\sup _{n \in \mathbb{N}}\left\|y^{(n)}\right\|_{b v}<\infty$ and any monotonicly increasing sequence $\left(n_{k}\right)$ of integers there exists a subsequence $\left(m_{k}\right)$ of $\left(n_{k}\right)$ with $\sum_{j=1}^{\infty} x y^{\left(m_{\jmath}\right)} \in E$ (pointwise sum).

- $E$ has the uniform gliding hump property (u_ghp) if the sequence $\left(m_{k}\right)$ in the definition of the p_ghp may be chosen independently of $x \in E$.

- $E$ has the pointwise weak gliding hump property (p_wghp) if the definition of the p_ghp is fulfilled for each 1-block sequence.

- $E$ has the uniform weak gliding hump property (u_wghp) if the definition of the u_ghp is fulfilled for each 1-block sequence.

We say that $E$ has the strong p ghp (u_ghp, p_wghp or u_wghp) if $\sum_{j=1}^{\infty} x y^{(m,)} \in E$ (pointwise sum) holds for any subsequence of $\left(m_{k}\right)$ in the above definitions; in this case, we use the notation sp_ghp, su ghp, sp_wghp and su_wghp, respectively.

REMARKS 3.2 . Let $E$ be a sequence space containing $\varphi$.

(a) Obviously, the definition of the ghp corresponds with the definition given in [20],[4] and the definition of the p_wghp corresponds to the weak gliding hump property considered by D. Noll [14].

(b) $E$ has the u.ghp if and only if $\mathcal{M}(E)$ has the ghp.

(c) su_ghp $\Longrightarrow$ su_wghp $\Longrightarrow$ u_wghp $\Longrightarrow$ p_wghp;

su_ghp $\Longrightarrow$ sp_ghp $\Longrightarrow$ sp_wghp $\Longrightarrow$ p_wghp;

su_ghp $\Longrightarrow u_{\text {ghp }} \Longrightarrow$ p_ghp $\Longrightarrow$ p_wghp; 
sll_wghp $\Longrightarrow$ sp_wghp and sp_ghp $\Longrightarrow$ p_ghp.

(In the last section we provide examples to show that most of these implications are strict.)

(d) Vach solid space has the su ghp and ach monotone space has the su_wghp. (Note. each solid sequence space is monotonc.)

(e) Examples of spaces $l:$ such that $\mathcal{M}(E)$ has the ghp may be found in [1, Remark 1].

(f) In [.1] T. Leiger and the first author proved the validity of theorems of Mazur Orlicz, type under the assumption that $M$ is a sequence space such that $\mathcal{M}(M)$ has the ghp, that is, $M$ has the 1 _ghp. Actually. in each instance only the fact that $\mathcal{M}(M)$ has the p_ghp was used in the arguments.

TIIEOREM 3.3. Let $E$ be an $F K$ space containing $\varphi$. Then $S_{E}$ has the st rong phlp; in particular, if $E$ is an $F K-A K$ space then $E$ has the strong p-ghp.

PROOF. The $F K$ topology of $F$ may be generated by seminorms

$$
p_{r}(r \in \mathbb{N}) \text { such that } p_{r}(x) \leq p_{r+1}(x) \quad(r \in \mathbb{N} \text { and } x \in E)
$$

Since $S_{E}$ is an $F K^{\circ}-A K^{\circ}$ space we may assume that $E$ is an $F K-A K^{-}$-space.

Now, let $x \in E$; be given. Then

$$
\sup _{\nu \geq n} p_{r}\left(\sum_{k=n}^{\nu} x_{k} e^{k}\right) \longrightarrow 0 \quad(n \rightarrow \infty \text { and } r \in \mathbb{N}) .
$$

Further let $\left(y^{(\jmath)}\right)$ be (a subsequence of) any block sequence satisfying $M:=\sup _{\jmath \in \mathbb{N}}\left\|y^{(\jmath)}\right\|_{b v}<\infty$. There exist index sequences $\left(\nu_{\jmath}\right)$ and $\left(\mu_{\jmath}\right)$ such that $\nu_{\jmath} \leq \mu_{j}<\nu_{\jmath+1} \quad(j \in \mathbb{N})$ and

$$
y^{(\jmath)}=\sum_{k=\nu}^{\mu,} y_{k}^{(\jmath)} e^{k}, \text { thus } y_{k}^{(\jmath)}=0 \text { for } k \notin\left[\nu_{\jmath}, \mu_{\jmath}\right] \text {. }
$$

On account of $(\circ)$ it is sufficient to prove $x y^{(\jmath)} \longrightarrow 0$ in $E$. For that end let $r \in$ IN be given. Then we have

$$
\begin{aligned}
p_{r}\left(x y^{(\jmath)}\right)=p_{r}\left(\sum_{k=\nu,}^{\mu,} x_{k} y_{k}^{(j)} e^{k}\right) & \leq \sup _{K \geq \nu,} p_{r}\left(\sum_{k=\nu,}^{K} x_{k} e^{k}\right) \sum_{k=\nu,}^{\mu,}\left|y_{k}^{(\jmath)}-y_{k+1}^{(\jmath)}\right| \\
& \leq M \sup _{K \geq \nu,} p_{r}\left(\sum_{k=\nu,}^{K} x_{k} e^{k}\right) \stackrel{\jmath \rightarrow \infty}{\longrightarrow} 0
\end{aligned}
$$

by $(*)$ which proves $x y^{(\jmath)} \longrightarrow 0$ in $E$.

REMARK 3.4. In general, $W_{E}$ fails the p_wghp. [Example: Let $\Sigma$ be the summation matrix and $E:=c_{\Sigma^{-1}}$. Then $W_{E}$ fails the p_wghp since $x:=e=\sum_{j} e^{\jmath} \in W_{E}$ (pointwise sum) and $(n)=,(2 j)$ does not have any subsequence $\left(m_{k}\right)$ such that $\tilde{x}:=\sum_{k} e^{m_{k}}$ (pointwise sum) $\in E$ since $\Sigma^{-1} \tilde{x} \in m_{0} \backslash c$.]

THEOREM 3.5. Let $E$ be a sequence space containing $\varphi$, and let $B$ be a matrix such that $E \subset c_{B}$. Then $E \subset S_{B}$ if $E$ has the p-wghp.

PROOF. Suppose $E$ has the p_wghp. We know from Theorem 6 of D. Noll [14] and Remark 3.2(a) that $\left(E^{\beta}, \sigma\left(E^{\beta}, E\right)\right)$ is weakly sequentially complete. Therefore, by an inclusion theorem of $\mathrm{G}$. Bennett and N. J. Kalton [2, Theorem 5] we get $E \subset W_{B}$, in particular $E \subset L_{B}$ and $E \subset \Lambda_{B}^{1}$.

Now, assume $E \subset W_{B}$ and $E \not \subset S_{B}$, that is, there exists an $x \in E \subset W_{B}=L_{B} \cap \Lambda_{B}^{1}$ with $x \notin S_{B}$, thus

$$
\lim _{B} x=\sum_{k=1}^{\infty} b_{k} x_{k}, \quad \sup _{n, r \in \mathbb{N}}\left|\sum_{k=1}^{r} b_{n k} x_{k}\right|<\infty \quad \text { and } \quad \sup _{\substack{n, r \in \mathbb{N} \\ r \geq \nu}}\left|\sum_{k=\nu}^{r} b_{n k} x_{k}\right| \not 0 .
$$


Therefore we may choose an $\eta>0$ and index sequences $\left(n_{j}\right),\left(\phi_{j}\right)$ and $\left(n_{j}\right)$ with $\alpha_{j} \leq, b_{\text {, such that }}$

$$
\left|\sum_{k=n}^{n} b_{n, k} x_{k}\right| \geq \eta \quad \text { for anch }, \in \mathbb{N}
$$

Now we emplov a gliding hump argument. Let $k_{0}:=1$ and choose $n_{i}^{*}$ such that

$$
\sum_{k=1}^{k_{1}}\left|b_{n k}-b_{k}\right|\left|x_{k}\right|<2^{-1} \quad\left(n \geq n_{1}^{*}\right)
$$

Then there exist a $\jmath_{1} \in \mathbb{N}$ with $n_{j_{1}}>n_{1}^{*}$ and a $k_{1}>\beta_{j_{1}}$ such that (note $x \in I_{B}$ )

$$
\max _{\substack{k \geq k_{1} \\ p \in \mathbb{N}_{0}}}\left\{\left|\sum_{k=k}^{\kappa+p} b_{n k} \cdot x_{k}\right|,\left|\sum_{k=k}^{k+p} b_{k} \cdot x_{k}\right|\right\}<2^{-1} \quad\left(n \leq n_{J_{1}}\right) .
$$

('hoose $n_{2}^{*} \geq n_{\jmath_{1}}$ such that

$$
\sum_{k=1}^{k_{1}}\left|b_{n k}-b_{k}\right|\left|x_{k}\right|<2^{-2} \quad\left(n \geq n_{2}^{*}\right)
$$

and a $k_{2}>k_{1}$ such that

$$
\max _{\substack{K>k_{2} \\ p \in \mathbf{N}_{0}}}\left\{\left|\sum_{k=K}^{K+r} b_{n k} x_{k}\right| \cdot\left|\sum_{k=\boldsymbol{k}}^{h+p} b_{k} x_{k}\right|\right\}<2^{-2} \quad\left(n \leq n_{2}^{*}\right) .
$$

Proceeding inductively, we get index sequences $\left(k_{\nu}\right),\left(J_{\nu}\right),\left(n_{\nu}\right)$ with

$$
n_{1}^{*}<n_{\jmath_{1}}<n_{2}^{*}<n_{3}^{*}<n_{\jmath_{2}}<\ldots<n_{2 \nu-1}^{*}<n_{j_{\nu}}<n_{2 \nu}^{*}<n_{2 \nu+1}^{*}<\ldots
$$

and

$$
k_{0}<\alpha_{\jmath_{1}} \leq \beta_{\jmath_{1}}<k_{1}<k_{2}<n_{\jmath_{3}}<\ldots<k_{2 \nu-2}<\alpha_{J_{\nu}} \leq \beta_{j_{\nu}}<k_{2 \nu-1}<k_{2 \nu}<\ldots
$$

fulfilling

$$
\sum_{k=1}^{k_{\nu-1}}\left|b_{n k}-b_{k}\right|\left|x_{k}\right|<2^{-\nu} \quad\left(n \geq n_{\nu}^{*}\right)
$$

and

$$
\max _{\substack{K \geq k, p \in \mathbf{N}_{0}}}\left\{\left|\sum_{k=K}^{K+p} b_{n k} x_{k}\right|,\left|\sum_{k=K}^{K+p} b_{k} x_{k}\right|\right\}<2^{-\nu} \quad\left\{\begin{array}{ll}
\text { if } \nu & \text { is odd and } n \leq n_{j_{\nu}} \\
\text { if } \nu \text { is even and } n \leq n_{\nu}^{*}
\end{array} .\right.
$$

Now, we define a subsequence $\left(y^{(\nu)}\right)$ of a 1-block sequence by

$$
y_{k}^{(\nu)}:= \begin{cases}1 & \text { if } \alpha_{\jmath_{\nu}} \leq k \leq \beta_{\jmath_{\nu}} \\ 0 & \text { otherwise }\end{cases}
$$

and consider

$$
y x \text { where } y:=\sum_{\nu=1}^{\infty} y^{(\nu)} \text { (pointwise sum) }
$$

Since $E$ has the p-wghp we may assume that $y x \in E$ (otherwise we switch over to a subsequence $\left(y^{\left(n_{k}\right)}\right.$ ) and adapt the chosen index sequences). For a proof of Theorem 3.5 it is sufficient to prove $y x=: z \notin c_{B}$. For this let $\nu \geq 2$ and $n:=n_{\jmath_{\nu}}$. Then (note, $\sum_{k} b_{k} z_{k}$ exists)

$$
\begin{aligned}
& \left|\sum_{k=1}^{\infty} b_{n k} z_{k}-\sum_{k=1}^{\infty} b_{k} z_{k}\right| \\
& \geq-\sum_{k=1}^{k_{2 \nu-2}}\left|b_{n k}-b_{k}\right|\left|x_{k}\right|+\left|\sum_{k=\alpha_{J_{\nu}}}^{\beta_{J_{\nu}}} b_{n k} x_{k}\right|-\sum_{r=\nu+1}^{\infty}\left|\sum_{k=\alpha_{j r}}^{\beta_{j r}} b_{n k} x_{k}\right| \\
& \quad \geq \eta-2^{-\nu}-2^{-\nu} \longrightarrow \eta>0 \text { for } \nu \rightarrow \infty .
\end{aligned}
$$


Now let $\nu \geq 2$ and $n:=n_{z_{\nu}}^{*}$. Then (sec above)

$$
\begin{aligned}
\left|\sum_{k=1}^{\infty} b_{n k} z_{k}-\sum_{k=1}^{\infty} b_{k} z_{k}\right| & \leq \sum_{k=1}^{\infty}\left|b_{n k}-b_{k}\right|\left|x_{k}\right|+\sum_{r=\nu+1}^{\infty}\left|\sum_{k=v_{1 r}}^{\beta_{k r}} b_{n k} x_{k}\right| \\
& \leq 2^{-\nu}+2^{-\nu} \longrightarrow 0(\nu-\infty) .
\end{aligned}
$$

Altogether we have proved $y x \notin c_{B}$.

Now, an obvious question is whether the statement in Theorem 3.5 remains true if we replace the domain $c_{B}$ by any separable $F K$ space $F$ with $E \subset F$. A positive answer is a consequence of the following theorem.

TIIFOREM 3.6. Let $E$ be a sequence space containing $\varphi$. Then the following statements are equivalent:

(i) $\left(E, \tau\left(E, E^{13}\right)\right)$ is an $A K$-space and $E^{\beta}$ is $\sigma\left(E^{\beta}, E\right)$ sequentially complete.

(ii) If $F^{\prime}$ is any separable $F K^{-}$-space with $E \subset F$ then $E \subset S_{F}$.

(iii) If $A$ is any matrix with $E \subset c_{A}$ then $E \subset S_{A}$.

PROOF. The equivalence (i) $\Leftrightarrow$ (ii) is Theorem $6,(\mathrm{i}) \Leftrightarrow$ (ii) of G. Bennett and N. J. Kalton [2]. The implication (ii) $\Rightarrow$ (iii) is obviously valid since domains $c_{A}$ are separable $F K^{-}$-spaces.

We are going to prove (iii) $\Rightarrow$ (i). Let (iii) be valid. Then $E^{\beta}$ is $\sigma\left(E^{\beta}, E\right)$-sequentially complete by [2, Theorem 5 , (iv) $\Rightarrow$ (i)].

Assume, $\left(E, \tau\left(E, E^{\beta}\right)\right)$ is not $A K$. Thus, we may choose an $x \in E$ and an absolutely convex $\sigma\left(E^{\beta}, E\right)-$ compact subset $K$ of $E^{\beta}$ such that

$$
p_{K}\left(x^{[n]}-x\right) \nrightarrow 0(n \rightarrow \infty) \quad \text { where } \quad p_{K}(z):=\sup _{a \in K}\left|\sum_{k} a_{k} z_{k}\right| \quad(z \in E)
$$

Therefore we may choose an index sequence $\left(n_{\imath}\right)$ and a sequence $\left(a^{(z)}\right)$ in $K$ such that

$$
\left|\sum_{k=n_{i}+1}^{\infty} a_{k}^{(i)} x_{k}\right| \geq \eta>0 \quad(i \in \mathbb{N}) .
$$

Since $K$ is $\sigma\left(E^{\beta}, E\right)$-compact, $\sigma\left(E^{\beta}, E\right)$ and $\sigma\left(E^{\beta}, \varphi\right)$ coincide on $K$ and $\sigma\left(E^{\beta}, \varphi\right)$ is metrizable we may assume that $\left(a^{(2)}\right)$ is $\sigma\left(E^{\beta}, E\right)$-convergent to an $a \in K$. (Otherwise we switch over to a subsequence of $\left(a^{(\jmath)}\right)$.) If $A$ denotes the matrix given by

$$
a_{i k}:=a_{k}^{(i)} \quad(i, k \in \mathbb{N})
$$

then -in summability language- the last assumption tells us

$$
E \subset c_{A} \quad\left(\text { even } E \subset \Lambda_{A}^{\perp}\right)
$$

From (*) we get $x \notin S_{A}$ which contradicts the assumption that (iii) is true.

COROLLARY 3.7. Let $E$ be a sequence space containing $\varphi$ and $F$ be a separable $F K$-space with $E \subset F$. If $E$ has the p_wghp then $E \subset S_{F}$.

PROOF. Theorem 3.6 and 3.5.

COROLLARY 3.8. Let $Y$ be a sequence space and $E$ be an FK-space with $\varphi \subset Y \cap E$ and $B$ be a matrix with $Y \cap S_{E} \subset c_{B}$. Then $Y \cap S_{E} \subset S_{B}$ if $Y$ has the p-wghp.

The statement remains true if we replace $c_{B}$ by any separable FK-space $F$. 
PROOF. Corollary 3.7 and the fact that $Y \cap S_{E}$, has the p-wghp.

COROLLARY 3.9 . Let $E$ be a separable $\mathrm{FK}$ space containing $\varphi$ such that $S_{E} \varsubsetneqq W_{E}$. Then $W_{E}$ fails the p_wghp (whereas $S_{E}$ has the strong p_ghp).

PROOF. Theorem 3.3 and Corollary 3.7.

4. APPIICATIONS.

REMARK 4.1 . Let $A=\left(a_{n k}\right)$ be a matrix with $\varphi \subset c_{A}$ and let $x \in c_{A}$. Then

$$
x \in S_{A} \Longleftrightarrow \sum_{k=1}^{\infty} a_{n k} x_{k} \text { converges uniformly in } n \in \mathbb{N} \text {. }
$$

This observation gives us a short proof of the following theorem containing a Toeplitz-Silverman theorem.

THEOREM 4.2 (matrices being conservative for $c_{0}$ ). For matrices $A=\left(a_{n k}\right)$ the following statements are equivalent:
(a) $c_{0} \subset c_{A}$.
(b) $c_{0} \subset S_{A}$.
(c) $\varphi \subset c_{A}$ and $\|A\|:=\sup _{n \in \mathbb{N}} \sum_{k=1}^{\infty}\left|a_{n k}\right|<\infty$.

PROOF. The implication (a) $\Rightarrow(\mathrm{b})$ comes from the $A K$-property of $c_{0}$ and the monotonicity of $F K$-topologies. (This statement follows also by Theorem 3.5 since $c_{0}$ obviously has the p_wghp.) Using standard estimations we may prove $(c) \Rightarrow(a)$. We are going to prove the essential part (b) $\Rightarrow(c)$.

Let $c_{0} \subset S_{A}$. Therefore, we can apply the above remark to any $x \in c_{0}$.

If $\|A\|=\infty$ we may choose a sequence $(n$,$) in \mathbb{I N}$ and index sequences $(\alpha$,$) and (\beta$,$) with \alpha, \leq \beta,<\alpha,+1$ $(j \in \mathbb{N})$ such that

$$
\sum_{k=\alpha}^{\beta,}\left|a_{n, k}\right| \geq j^{2} \quad(j \in \mathbb{N})
$$

Defining $y \in c_{0}$ by

$$
y_{k}:= \begin{cases}\frac{1}{j} \operatorname{sgn} a_{n, k} & \text { if } \alpha, \leq k \leq \beta \\ 0 & \text { otherwise }\end{cases}
$$

we get

$$
\left|\sum_{k=\alpha,}^{\beta,} a_{n, k} y_{k}\right|=\frac{1}{j} \sum_{k=\alpha}^{\beta_{3}}\left|a_{n, k}\right| \geq j \quad(j \in \mathbb{N}) .
$$

Thus $\sum_{k=1}^{\infty} a_{n k} y_{k}$ does not converge uniformly in $n \in \mathbb{I N}$ which contradicts $c_{0} \subset S_{A}$.

Using the same method we get also a proof of a theorem containing a theorem of Hahn (equivalence of (a) and (c)). However, we should mention that the proof of '(a) $\Longrightarrow$ (c)' presented in [18, Theorem 4.1, p. 110] is more elegant.

THEOREM 4.3 (matrices summing each absolute summable sequence). For matrices $A=\left(a_{n k}\right)$ the following statements are equivalent:
(a) $\ell \subset c_{A}$.
(b) $\ell \subset S_{A}$.
(c) $\varphi \subset c_{A}$ and $\sup _{n, k \in \mathbb{N}}\left|a_{n k}\right|<\infty$. 
PROOF: (a) $\Rightarrow$ (b) follows from the continuity of the inclusion map and the fact that $\ell$ is an $F \boldsymbol{K} .1 \boldsymbol{K}$ space and the monotonicity of $F K$ topologies whereas $(c) \Rightarrow($ a $)$ may be proved with classical estimations. '(b) $\Rightarrow(c)$ ': Let $\ell \subset S_{A}$. Thus, obviously, $\varphi \subset c_{A}$ is true. Wo assume $\sup _{n \in \mathbb{N}}\left|a_{n k}\right|=\infty$. Then we may. choose sequences $\left(n_{j}\right)$ and $(k$,$) in \mathbb{I N}$ with $k,<k_{j+1}(j \in \mathbb{N})$ such that

$$
\left|a_{n, k}\right| \geq J^{2} \quad(J \in \mathbb{N})
$$

Defining $y=\left(y_{k}\right)$ by

$$
y_{k}:= \begin{cases}\frac{1}{j^{2}} \operatorname{sgn} a_{n, k}, & \text { if } k=k, \\ 0 & \text { otherwise }\end{cases}
$$

we obviously get

$$
\left|\sum_{k=k}^{k} a_{n, k} y_{k}\right|=\left|a_{n, k},\right| \frac{1}{\jmath^{2}} \geq 1 \quad(\jmath \in \mathbb{N}) .
$$

The last estimation gives us $y \notin S_{A}$ that contradicts $\ell \subset S_{A}$.

In the next step we use this method to reprove both the well-known Schur theorem and the Hahn theorem. (The Schur theorem characterizes the matrices summing all bounded sequences, the Hahn theorem tells us that a conservative matrix which sums all $x \in \chi$ sums also all bounded sequences where $\chi$ denotes the set of all sequences with 0 and 1.) Moreover, we take an extended version of Schur's theorem (see [3]) into consideration.

THEOREM 4.4 (Extended theorem of Schur, theorem of Hahn). Let $A=\left(a_{n k}\right)$ be a matrix. Then the following statements are equivalent:

(a) $m \subset c_{A}$.

$\left(\mathrm{a}^{*}\right) \quad m \subset S_{A}$.

(b) $\exists \mu=\left(\mu_{k}\right), 0<\mu_{k} \nearrow \infty: m_{\mu} \subset c_{A}$.

(b*) $\exists \mu=\left(\mu_{k}\right), 0<\mu_{k} \nearrow \infty: m_{\mu} \subset S_{A}$.

(c) $\chi \subset c_{A}$, that is $m_{0} \subset c_{A}$.

(c*) $\chi \subset S_{A}$, that is $m_{0} \subset S_{A}$.

(d) $\varphi \subset c_{A}$ and $\sum_{k=1}^{\infty}\left|a_{n k}\right|$ converges uniformly in $n \in \mathbb{N}$.

$\left(\mathrm{d}^{*}\right) \quad c_{0} \subset c_{A}$ and $\limsup \sum_{k=1}^{\infty}\left|a_{n k}-a_{k}\right|=0$ where $a_{k}$ denotes the limit of the $k$-th column.

(e) $\varphi \subset c_{A}$ and $\exists \mu=\left(\mu_{k}\right), 0<\mu_{k} \nearrow \infty: \sum_{k=1}^{\infty} \mu_{k}\left|a_{n k}\right|$ converges uniformly in $n \in \mathbb{N}$.

$\left(\mathrm{e}^{*}\right) \quad c_{0} \subset c_{A}$ and $\exists \mu=\left(\mu_{k}\right), 0<\mu_{k} \nearrow \infty: \limsup _{n} \sum_{k=1}^{\infty} \mu_{k}\left|a_{n k}-a_{k}\right|=0$.

Thereby, we can choose in (b), ( $\left.b^{*}\right)$ and (e) a common sequence $\mu$.

REMARK 4.5. Originally, Schur proved '(a) $\Leftrightarrow\left(\mathrm{d}^{*}\right)^{\prime}$ and $\lim _{A} x=\sum_{k=1}^{\infty} a_{k} x_{k}(x \in m)$ if $(\mathrm{a})$ or $\left(\mathrm{d}^{*}\right)$ in 4.4 is valid.

In case of conservative matrices the equivalence $(a) \Leftrightarrow(c)$ is Hahn's theorem.

PROOF of 4.4. We are going to check the following chain of implications:

$$
(\mathrm{b}) \stackrel{(1)}{\Rightarrow}\left(\mathrm{b}^{*}\right) \stackrel{(2)}{\Rightarrow}\left(\mathrm{a}^{*}\right) \stackrel{(3)}{\Rightarrow}(\mathrm{a}) \stackrel{(4)}{\Rightarrow}(\mathrm{c}) \stackrel{(5)}{\Rightarrow}\left(\mathrm{c}^{*}\right) \stackrel{(6)}{\Rightarrow}(\mathrm{d}) \stackrel{(7)}{\Rightarrow}\left(\mathrm{d}^{*}\right) \stackrel{(8)}{\Rightarrow}\left(\mathrm{e}^{*}\right) \stackrel{(9)}{\Leftrightarrow}(\mathrm{e}) \stackrel{(10)}{\Rightarrow}(\mathrm{b}) \text {. }
$$

The implications (2), (3) and (4) and the equivalences (7) and (9) are obviously true.

The implications (1) and (5) are immediate corollaries of Theorem 3.5 since $m_{\mu}$ and $m_{0}$ have the p_wghp. 
For a proof of $(8)$ and (10) we refer to [3].

Now, we give a proof of (6). For that we assume that $A$ is a matrix with real entries. [In the general case of complex entries we have to note that $\sum_{k}\left|a_{n k}\right|$ converges uniformly in $n \in \mathbb{I N}$ if and only if this is true for the real part of $a_{n k}$ and the imaginary part of $a_{n k}$.]

Let $\left(c^{*}\right)$ be true. Then $\varphi \subset c_{A}$.

If $\sum_{k=1}^{\infty}\left|a_{n k}\right|$ does not converge uniformly in $n \in \mathbb{N}$ then we may choose an $\eta>0$, a sequence $(n$,$) in \mathbb{N}$ and index sequences $\left(\alpha_{\jmath}\right)$ and $\left(\beta_{\jmath}\right)$ with $\alpha, \leq \beta,<\alpha_{\jmath}$ such that

$$
\sum_{k=\alpha}^{\beta}\left|a_{n, k}\right| \geq \eta \quad(\jmath \in \mathbb{N}) .
$$

We define $y \in m_{0}$ by

$$
y_{k}:= \begin{cases}\operatorname{sgn} a_{n, k} & \text { if } \alpha, \leq k \leq \beta \\ 0 & \text { otherwise }\end{cases}
$$

Since

$$
\left|\sum_{k=\alpha}^{\beta,} a_{n, k} y_{k}\right|=\sum_{k=\alpha}^{\beta,}\left|a_{n, k}\right| \geq \eta \quad(j \in \mathbb{N})
$$

the series $\sum_{k=1}^{\infty} a_{n k} y_{k}$ does not converge uniformly in $n \in \mathbb{N}$. Therefore $y \notin S_{A}$ which contradicts $\chi \subset S_{A}$.

\section{EXAMPLES.}

The aim of this section is the presentation of some examples distinguishing almost all of the gliding hump properties. For that purpose we collect known connections between gliding hump and related properties of sequence spaces in the following graphic.

Figure 1:

Each arrow stands for 'implies' and the corresponding number in the circle gives the number of the example in 5.1 proving the strictness of the implication.

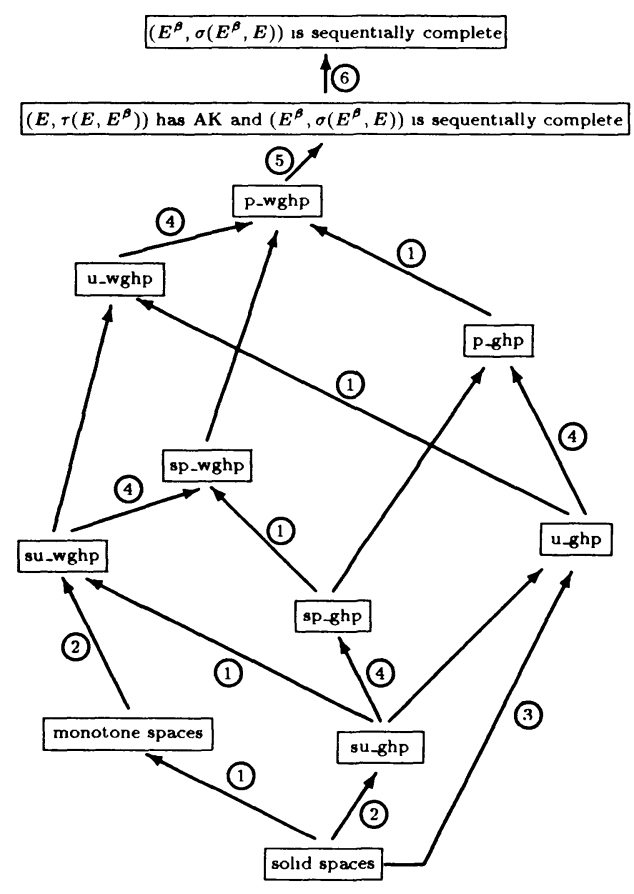


EXAMPIES 5.1. (1) $m_{0}$ is a monotone space, thus it has all of the weak gliding hump properties ?". wghp. However. it does not have the p.ghp, thus no??-ghp and it is not solid.

(2) The sequence space $f_{0}$ of all sequences almost convergent to 0 has all of the gliding hump properties. Furthermore, it is not a monotone space.

For a proof of the first statement we may prove that $f_{0}$ has the su_ghp by modifying Sinyders proof of [20, Theorem7].

(3) $E:=\ell_{\mu} \cap\left(c_{0}\right)_{(c}$, with $\mu_{k}=k^{2}(k \in \mathbb{N})$ has the u-ghp since $\mathcal{M}(E)$ has the gliding hump property (see [23, Theorem 3.3 and 3.1$]$ ). We don't know whether $E$ has the su_ghp. Therefore, it may be a candidate to distinguish the properties su_ghp and $u_{-g h p .}$

(4a) $E:=\ell_{2} \cap c . s$ has the sp-ghp (thus all ?p_?ghp) since it is an $F K^{\circ} A K^{\circ}$-space (Theorem 3.3). Furthermore, with $\left[17\right.$, Corollary 4.4] we get that $E$ is a sum space. Thus, by definition of a sum space $\mathcal{M}\left(E^{\prime}\right):=E^{\prime}$. Therefore, $\mathcal{M}\left(E^{\prime}\right)=E^{\jmath}=\ell_{2}{ }^{\prime}+c s^{\jmath}=\ell_{2}+b v \subset c$. From this and the fact that $e \in \mathcal{M}(E)$ we may derive that $E$ cannot have the u_wghp (thus ?u_?ghp).

(4b) Considering the James space we get further sequence spaces having the same gliding humps properties as the example in (4a). For that let $\omega$ be the space of all real sequences and let

$$
N(x)=\sup \left[\sum_{i=1}^{n}\left(x_{p_{2 t-1}}-x_{p_{2 t}}\right)^{2}+x_{p_{2 n+1}}^{2}\right]^{\frac{1}{2}}
$$

where the supremum is taken over all positive integers $n$ and all finite increasing sequences of integers $p_{1}, \ldots, p_{2 n+1}$. Then

$$
S_{N}=\{x \in \omega \mid N(x)<\infty\}
$$

(together with its natural norm $N$ ) is a $B K$-space and the closure $J=S_{N}^{0}$ of $\varphi$ in $S_{N}$ is called James space (see [16]). We'll make use of the following facts:

(i) $S_{N}$ is a BK-algebra with identity $e$.

(ii) $J=S_{N} \cap c_{0}$

(iii) $\left(e^{k}, E_{k}\right)$ is a shrinking basis for $J^{f}$ so, in particular, $J^{f}$ is $A K$ thus $A D$.

(iv) $\quad S_{N}=J \oplus\langle e\rangle=J^{f S}$.

Now by (i) and (iv) we get $\mathcal{M}\left(J^{f f}\right)=J^{f f}$ and by (iii) we get $\mathcal{M}\left(J^{f}\right)=\mathcal{M}\left(J^{f f}\right)$ (see [7, Proposition 3.4]). Therefore by (ii) and (iv) we have

$$
\mathcal{M}\left(J^{f}\right)=\mathcal{M}\left(J^{f f}\right)=J^{f f}=J \oplus\langle e\rangle \subset c .
$$

As in (4a) we conclude that $J^{f}$ has the sp_ghp (thus all ?p_?ghp) since it is an $F K$ - $A K$-space. Furthermore, by (*) and $e \in \mathcal{M}\left(J^{f}\right)$ we get that $J^{f}$ cannot have the u_wghp (thus ?u_?ghp).

From (*) we know that $J^{f}$ is a sum space. Thus by [17, Corollary 4.4] $J^{f} \cap E$ will be a sum space too if $E$ is any $F K$-space with unconditional basis $\left(e^{k}\right)$. Then

$$
\mathcal{M}\left(J^{f} \cap E\right)=\left(J^{f} \cap E\right)^{f}=J^{f f}+E^{f}=(J \oplus\langle e\rangle)+E^{f}
$$

Now, let $E$ be any $F K$-space with unconditional basis $\left(e^{k}\right)$ such that $E^{f} \subset c$. Then, as above we may conclude, $J^{f} \cap E$ has all ?p_?ghp and no ?u_?ghp. 
(5) Obviously. bs does not have the p_wghp thus none of the gliding hump properties in consideration. However it is known that $\left(b . s, \tau\left(b . s, b r_{0}\right)\right)$ is an $A h$ space and $\left(b r_{0}, \sigma\left(b v_{0}, b s\right)\right)$ is sequentially complete.

(6) Let $F$ be any separable $F K$ space with $S_{F} \subsetneq W_{F}$ (for example, the domain of a conull matrix being not strongly comull) and let $E:=W_{F}$. Then $\left(E^{3}, \sigma\left(E^{3}, E\right)\right.$ ) is sequentially complete (see [1, Theorem 1 and 2]) but $\left(E, \tau\left(K, V^{* 3}\right)\right)$ is not an $A K$-space since otherwise from Theorem 3.6 we would get $S_{F} \supset V_{F}=H_{F}$ thus $S_{F}=W_{F}$.

Closing the paper we mention, that we don't know whether there is a difference between the s?_?ghp and the corresponding ?.?ghp (see Figure 1 and lixample 5.1(3)).

\section{REFERENCES}

1. BFNNETT, G. Sequence spaces with small $\beta$-duals, Math. Z. 194 (1987), $321 \cdot 329$.

2. BENNETT, G., KALTON, N. J. Inclusion theorems for K-spaces, Canad. J. Math. $\underline{25}$ (1973), $511-524$.

3. BOOS, J. Eine Erweiterung des Satzes von Schur, Manuscripta Math. $\underline{31}$ (1980), 111-117.

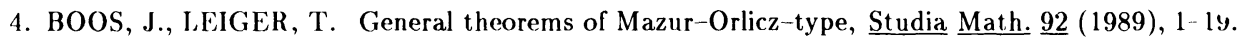

5. BOOS, J., LEIGER, T. Consistency theory for operator valued matrices, Analysis 11 (1991), 279-292.

6. BRUDNO, A. L. Summation of bounded sequences by matrices, Mat. Sb. (N. S.) 16 (1945), 191-247. (Russian; english abstract).

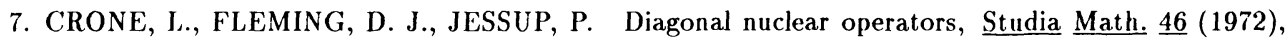
51-56.

8. JAKIMOVSKI, A., LIVNE, A. On matrix transformations between sequence spaces, J. Analyse Math. 25 (1972), 345-370.

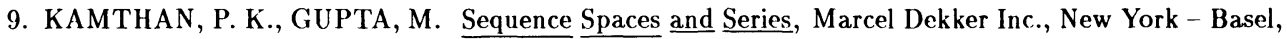
1981.

10. KÖTHE, G. Topological Vector Spaces I, Springer, Berlin-Heidelberg-New York, 1969.

11. KÖTHE, G., TOEPLITZ, O. Lineare Räume mit unendlich vielen Koordinaten und Ringe unendlicher Matrizen, J. reine und angew. Math. 171 (1934), 193-226.

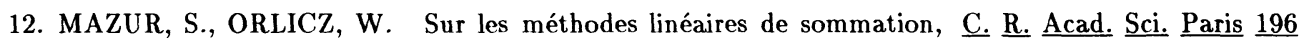
(1933), 32-34.

13. MAZUR, S., ORLICZ, W. On linear methods of summability, Studia Math. 14 (1955), 129-160.

14. NOLL, D. Sequential completeness and spaces with the gliding humps property, Manuscripta Math. $\underline{66}(1990), 237-252$.

15. NOLL, D., STADLER, W. Sliding hump technique and spaces with the Wilansky property, Amer. Math. Soc. 105 (1989), 903-910.

16. RUCKLE, W. H. The construction of sequence spaces that have a Schauder basis, $\underline{\text { Can. }} \underline{\mathrm{J}} . \underline{\text { Math. }} \underline{18}$ (1966), 1281-1293.

17. RUCKLE, W. H. An abstract concept of the sum of a numerical series, Can. $\underline{\text { J. Math. }} \underline{72}$ (1970), 863-874.

18. RUCKLE, W. H. Sequence Spaces. Pitman Advanced Publishing Program, Boston - London Melbourne, $198 \overline{1 .}$

19. SCHUR, J. Über lineare Transformationen in der Theorie der unendlichen Reihen, $\underline{\text { J. reine }} \underline{\text { angew. }}$ Math. 151 (1920), 79-111.

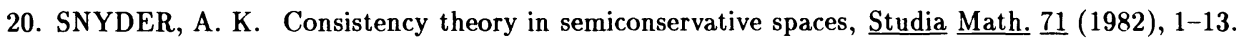

21. STADLER, W. Zu einer Frage von A. Wilansky, Arch Math. $\underline{48}$ (1987), 149-152. 
22. TOEPIIT\%, O. Über allgemeine lineare Mittelbildungen, Prace mat. fiz. 22 (1911), 113118.

23. T\%IMBBALARIO, J. Some Mazur Orlic\% Brudno like theorems, Studia Math. 18 (1973), 107 117.

21. WILANSKY, A. Summability through Functional Analysis, volume 85 of Notas de Matemática, North Ilolland, Amsterdam New York Oxford. 19x.1. 


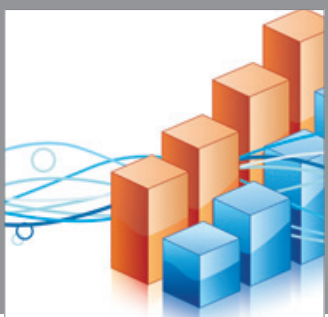

Advances in

Operations Research

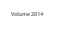

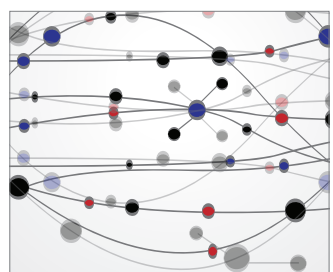

\section{The Scientific} World Journal
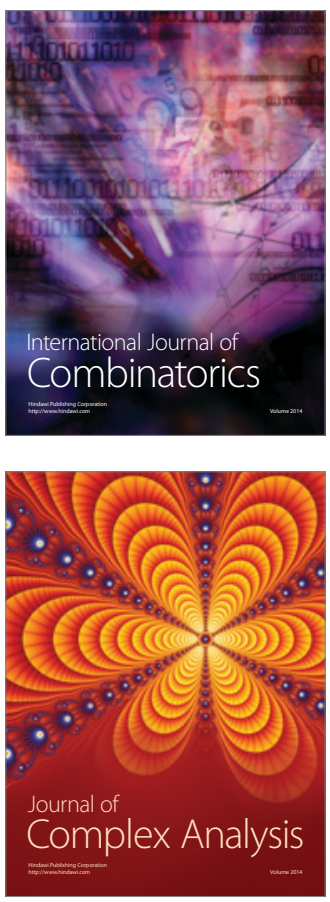

International Journal of

Mathematics and

Mathematical

Sciences
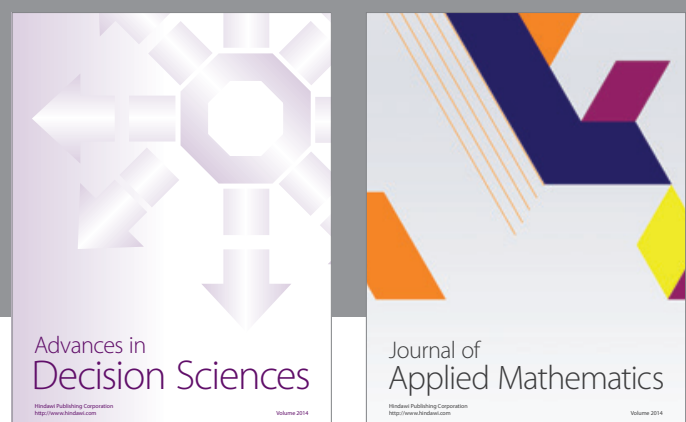

Journal of

Applied Mathematics
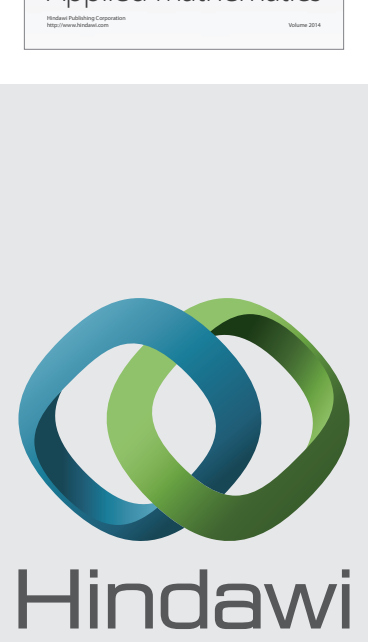

Submit your manuscripts at http://www.hindawi.com
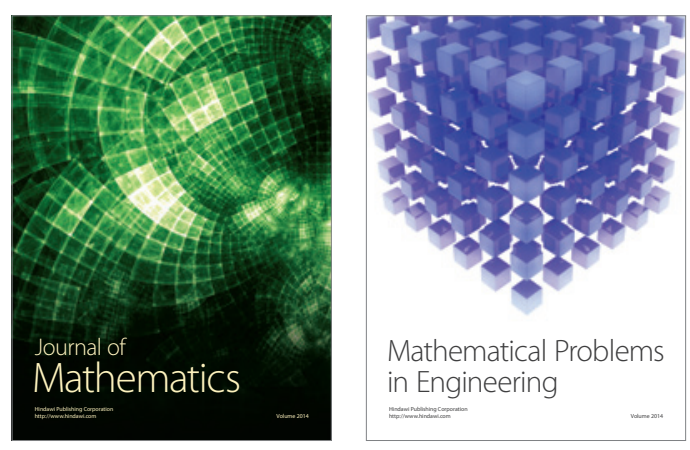

Mathematical Problems in Engineering
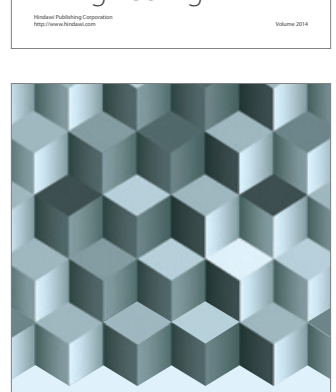

Journal of

Function Spaces
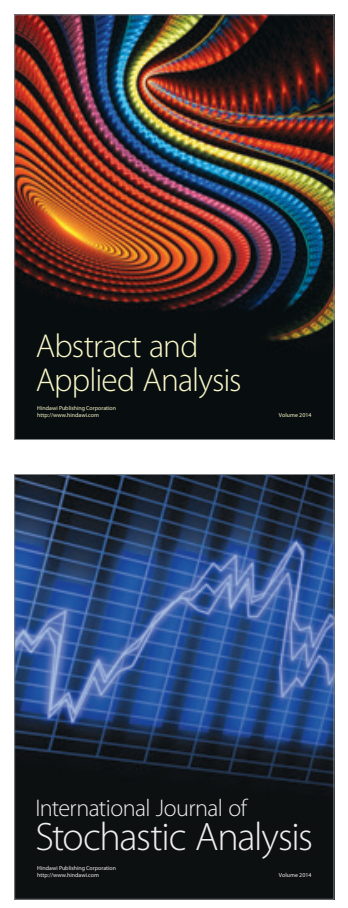

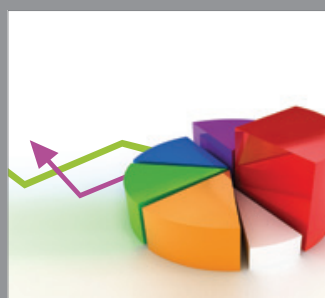

ournal of

Probability and Statistics

Promensencen
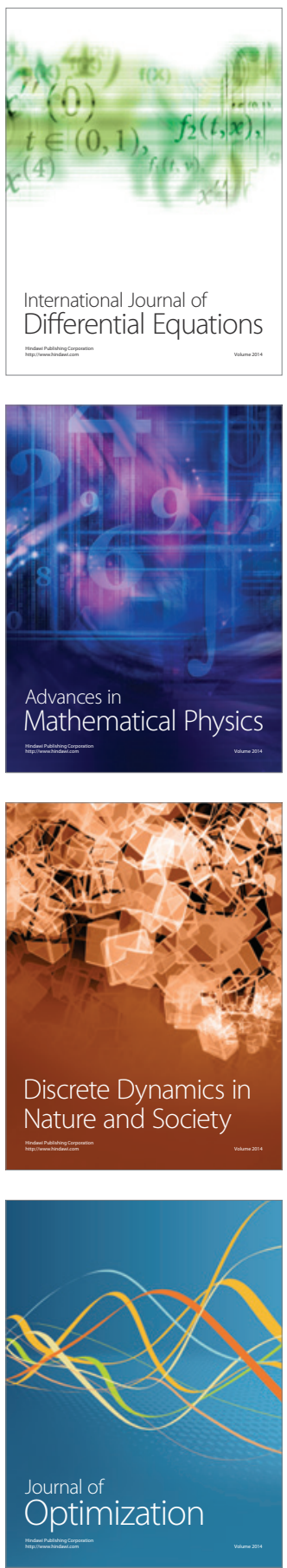